\title{
Phototimer setup for CR imaging
}

Emmanuel G. Christodoulou, ${ }^{\text {a) }}$ Mitchell M. Goodsitt, and Heang-Ping Chan

Department of Radiology, University of Michigan Medical Center, 1500 East Medical Center Drive, Ann Arbor, Michigan 48109-0030

Thomas W. Hepburn

12361 Duffield Road, Gaines, Michigan 48436

(Received 20 October 1999; accepted for publication 28 August 2000)

\begin{abstract}
A study was performed to investigate the feasibility of using the standard deviation $(\sigma)$ of the pixel values in a computed radiography $(\mathrm{CR})$ image and a measure of the median incident exposure on the imaging plate (IP) as parameters for setting up phototimers in a CR system. Slabs of Lucite ${ }^{\mathrm{TM}}$ 4-, 6-, and 8-in.-thick were imaged with a CR system at 70, 90, and $125 \mathrm{kVp}$ at various $\mathrm{mA} s$ values both with grid and without grid. Incident IP exposures were measured with an ionization chamber. Images were analyzed on a workstation. The $\sigma$ 's in the "flat field" images were found to be approximately related to the mean incident exposure $E$ by the relationship: $\sigma \propto E^{-1 / 2}$, indicating the quantum-noise-limited operation of the system. Derived relationships between the reading sensitivity of the (IP) reader ( $S$ number) and $\sigma$ can be used to obtain images with a specific noise level. At our institution, where a 400 speed screen-film system is used for general radiography and 200 speed for chest radiography, radiologists generally find CR image quality acceptable when $\sigma \leqslant 11$ $(S \leqslant 400)$ for general radiography $(50-90 \mathrm{kVp})$, and $\sigma \leqslant 8(S \leqslant 200)$ for chest radiography (125 $\mathrm{kVp}$ ). However, factors other than the amount of $\mathrm{x}$-ray quanta that form the useful image, such as the image processing mode and the amount of collimation, may affect both the sensitivity value and the image quality. (C) 2000 American Association of Physicists in Medicine.
\end{abstract}

[S0094-2405(00)01811-3]

Key words: computed radiography, automatic exposure control, phototimer

\section{INTRODUCTION}

In film radiography, phototimers are used to yield approximately constant film optical density (OD) regardless of patient thickness or x-ray beam energy. The phototimers are adjusted so that the resulting film OD is near optimum for the specific clinical diagnosis. As computed radiography (CR) and other digital systems replace screen-film systems, it is desirable to investigate methods for adjusting phototimers to achieve optimum exposure on those imaging media. The phototiming parameter for CR is complicated by the fact that $\mathrm{CR}$ processing is designed to produce an approximately constant mean pixel value $(\sim 511)$ or gray level irrespective of x-ray exposure.

In screen-film systems the image is contrast limited, while in CR systems the image is typically noise limited because the system has a wide dynamic range, and the image contrast can be changed using digital transformations. ${ }^{1,2}$ Also, unlike screen-film, the image acquisition and display functions occur separately in $\mathrm{CR}{ }^{3}$ This enables CR systems to compensate for over- and underexposure of the imaging plate during display and to potentially lower the number of image retakes. ${ }^{4,5}$ However, underexposure results in noisy images and overexposure results in increased radiation burden to patients. Proper phototimer adjustment can potentially reduce these problems.

In the present work, we investigate a method to adjust phototimers when acquiring images with a CR system. For all CR systems, the photostimulable luminescent values that are generated at readout are directly related to the exposures incident upon the CR plates. Autoranging from improved display contrast is accomplished by these systems via analysis of histograms of the photostimulable luminescent values of the pixels which correspond to the energy deposited in the pixels. Each manufacturer defines an output parameter related to the mean or median exposure determined from these histograms, and these parameters may be used like the optical density of film for phototimer setup. The parameter employed by Fuji is termed the sensitivity number $(S)$. This number is determined from the median value $S_{k}$ of the histogram via the relationship $S=4 \times 10^{\left(4-S_{k}\right)}$, and is mapped to the median digital output value $511 .^{6}$ For the standard resolution imaging plates, the $S$ number is defined to be equal to 200 divided by the median exposure to the CR plate in $\mathrm{mR}$ for an $80 \mathrm{kVp} \mathrm{x}$-ray beam. ${ }^{5}$ The corresponding parameter for the Kodak CR system is termed the exposure index (EI), which is defined by ${ }^{7}$

$$
\begin{aligned}
\mathrm{EI}= & 2000+1000 \\
& \times \log _{10}\{\text { average exposure to plate in } \mathrm{mR}\} .
\end{aligned}
$$

Similarly, Agfa has defined a parameter termed the LgM that can be shown to be related to the median plate exposure by

$\operatorname{LgM}=2 \times \log _{10}(\mathrm{SAL})-3.9478$, 
where $\mathrm{SAL}=\left[1800^{2} \times(\right.$ speed class $/ 200) \times($ dose in $\mu \mathrm{Gy} / 20)$ $\times[\mathrm{IPF}]^{1 / 2}$, speed class is the user selected nominal speed of the CR plate (e.g., 200, 400, 600, etc.), and IPF is the imaging plate sensitivity correction factor $(\mathrm{IPF}=1$ for $\mathrm{MD} 10$ plate). ${ }^{8,9}$

In this paper, we propose a method for setting up phototimers using the Fuji $S$ number and the standard deviation $(\sigma)$ of the pixel values in a region of interest (ROI) from a "flat" field image. The latter is a measure of the noise present in the image. Since we only had access to a Fuji CR system, our results pertain directly only to that system. However, the principle of the method can be implemented with the other CR systems by using their plate exposure indicator parameters that correspond to the Fuji $S$ number.

\section{MATERIALS AND METHODS}

In this study, we used a $20 \mathrm{~cm} \times 25 \mathrm{~cm}$ Fuji ${ }^{\circledR}$ type ST-V CR plate (Fuji Medical Systems, Inc., Stamford, CT), chosen randomly from the pool of plates used routinely in our clinic. The plate was loaded in a Fuji imaging plate (IP) cassette. The same IP was used in the entire experimental procedure in order to eliminate variations in plate sensitivity as a source of error. The plate was exposed with a moving grid or without a grid. The grid had aluminum interspaces, a grid ratio of 12:1, and a strip density of 40 lines per $\mathrm{cm}$. In both the with-grid and without-grid cases, the incident exposures to the plate were maintained at approximately $0.5,1.0$, and 2.0 $\mathrm{mR}$. Three different beam qualities were employed: 70,90 , and $125 \mathrm{kVp}$. The HVL of the beam was $2.9 \mathrm{~mm}$ of $\mathrm{Al}$ at 80 $\mathrm{kVp}$. "Flat" field images of homogeneous acrylic (Lucite ${ }^{\mathrm{TM}}$ ) phantoms were obtained. Three different phantom thicknesses, 4, 6, and 8 in., were used for each beam quality and total exposure. A Keithley® Model 35050A dosimeter with a 15-cc ionization chamber (Keithley Instruments, Inc., Cleveland, $\mathrm{OH}$ ) was used to measure the exposure on the IP. The IP was processed in a calibrated Fuji ${ }^{\circledR}$ FCR 9000 plate reader with the Semi-automatic mode. In this mode the overall reading sensitivity ( $S$ number) is determined by analyzing the image histogram from a predetermined rectangular shaped subregion located at the center of the IP. Each image was processed using the "Test-Sensitivity" image acquisition menu, which employs a linear input-output transfer curve. For this particular mode, the $L$ (latitude) number, which is the logarithm of the useful range of exposures for analog-to-digital conversion, is fixed to be 2 (i.e., conversion range 100:1). The plate reader was interfaced to a Siemens ${ }^{\circledR}$ SIENET MagicView 1000 workstation (Siemens Medical Systems, Inc., Islen, NJ).

Figure 1 shows a schematic diagram of the irradiation geometry used in the with-grid and without-grid cases. The exposure to the plate was determined separately, as follows.

Without a grid, the plate exposures were measured with the presence of a loaded CR cassette. The cassette was positioned on the tabletop, and aligned so that the center of the $\mathrm{x}$-ray beam coincided with the center of the cassette. The ionization chamber was placed directly on top of the cassette

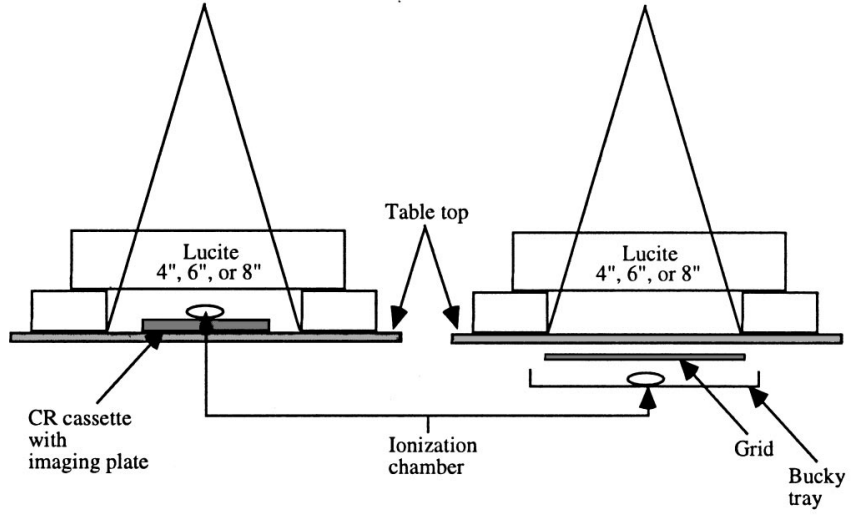

(a)

(b)

FIG. 1. Schematic diagram of the irradiation geometry (a) without a grid, and (b) with a grid (not to scale).

at the central position. The Lucite phantom was supported 3 in. above the tabletop.

With a grid, the plate exposures were measured without the presence of a loaded cassette, because the Bucky traygrid slot was not wide enough to accommodate the ionization chamber on top of the cassette. The ionization chamber was placed by itself in the Bucky tray. To determine the corresponding exposure with the presence of the loaded cassette, a set of measurements was performed using the following procedure: First, the Bucky tray was retracted from the table. Next, the grid was removed from its holder and supported above the Bucky tray at the same distance as in the normal Bucky tray-grid configuration. The tabletop was then moved laterally to a location on top of the grid. Then, two measurements were made - one with the ionization chamber centered on top of the cassette in the Bucky tray, and the other with only the ionization chamber centered in the Bucky tray. The ratio of the two measurements yielded a correction factor. This correction factor takes into account the difference between the backscatter with and without the cassette. The exposures determined without a cassette in the Bucky tray were multiplied by the correction factor to yield values in the presence of the cassette.

In both the with- and without-grid cases, seven exposures were made for each experimental condition (i.e., for each combination of $\mathrm{kVp}$, exposure level, and phantom thickness). The average of the seven exposures for a given condition was considered to be the exposure to the plate for that condition during the experimental procedure, which was performed without the presence of the ionization chamber. The reproducibility of the exposures was in the worst case within $2 \%$. The IP was processed 6 min after each exposure.

In each "flat field" image three circular ROIs were selected. One was located at the center of the image. The others were $2 \mathrm{~cm}$ to the left and right of the central ROI. The three ROIs were arranged in the direction perpendicular to the $\mathrm{x}$-ray tube anode-cathode axis in order to avoid exposure nonuniformity due to the "heel" effect. Each ROI contained about 10000 pixels. The average standard deviation of the pixel values in the three ROIs was computed and employed 
as the "standard deviation of the pixel values" for each experimental condition.

As stated previously, the $S$ number of a Fuji CR system is defined to be 200 divided by the median plate exposure in $\mathrm{mR}$ for an $80 \mathrm{kVp}$ x-ray beam. The dependence of the $S$ numbers on the spectrum of the $\mathrm{x}$-ray beam incident upon the plate has not been previously described. However, a fairly strong dependence would be expected since variations in $\mathrm{kVp}$ and filtration will produce $\mathrm{x}$-ray beams with effective energies that are below, at, or above the $37.4 \mathrm{keV}$ $K$-absorption edge of barium, which is a primary component of the CR plates. To account for this effect, we chose to employ the following generalized expression for $S$ numbers:

$$
S=\frac{c}{\bar{E}},
$$

where $c$ is a parameter that depends on $\mathrm{kVp}$ and phantom thickness. For uniform phantoms like the acrylic plates were employed, the median and the mean values $(\bar{E})$ of the incident exposure on the IP are the same. The mean exposure is the one that is measured experimentally. Therefore, we use the mean exposure in the equation. We estimated $c$ for each condition ( $\mathrm{kVp}$, phantom thickness, and grid/no grid) studied by applying linear least-squares fits of the three measured average plate exposures $(\bar{E})$ (approximately $0.5,1$, and 2 $\mathrm{mR}$ ) and the corresponding indicated $S$ numbers to

$$
\log (S)=\log (c)-\log (\bar{E}) \text {. }
$$

Since the pixel values in the CR images are normalized similar to those in CT and DSA, the noise level of the CR image is expected to be inversely related to the incident exposure on the IP (See Sec. IV). This noise level as represented by the standard deviation of the pixel values in the image, $\sigma$, can be expressed as

$$
\sigma=a \cdot \bar{E}^{b},
$$

where the parameters $a$ and $b$ depend on $\mathrm{kVp}$ and phantom thickness. The values of $a$ and $b$ for exposures with and without grid, for each $\mathrm{kVp}$ and phantom thickness, were estimated by linear least-squares fitting the experimental data to

$$
\log (\sigma)=\log (a)+b \log (\bar{E}) .
$$

\section{RESULTS}

Table I shows the values of the parameter $c$ of the generalized $S$-number equation [Eq. (1)] for various $\mathrm{kVp}$ and phantom thicknesses, both with and without a grid. Table II shows the values of $a$, the proportionality factor, and $b$, the exponent, for the noise versus plate exposure equation [Eqs. (3) and (4)]. The correlation coefficients for the fit range from 0.983 to 1.000 .

The variation of the $S$ numbers with $\sigma$, for different $\mathrm{kVp}$ values and phantom thicknesses, is shown in Figs. 2(a) and 2(b). To generate these plots, the fitted $a$ and $b$ values in Table II were employed in Eq. (3) to compute the mean plate exposures corresponding to integer noise values between $\sigma$
TABLE I. Dependence of the values of the parameter $c$ in equation $S \#=c /($ average exposure), on $\mathrm{kVp}$ and phantom thickness.

\begin{tabular}{cccc}
\hline \hline \multirow{2}{*}{$\begin{array}{c}\text { Lucite thickness } \\
\text { (in.) }\end{array}$} & $\begin{array}{c}c \\
\text { (with grid) }\end{array}$ & $\begin{array}{c}c \\
\text { (without grid) }\end{array}$ \\
\hline \multirow{2}{*}{70} & 4 & 146 & 203 \\
& 6 & 137 & 194 \\
90 & 4 & 142 & 187 \\
& 6 & 143 & 183 \\
125 & 8 & 144 & 185 \\
& 6 & 160 & 187 \\
& 8 & 165 & 188 \\
\hline \hline
\end{tabular}

$=8$ and $\sigma=11$. These mean exposures and the fitted $c$ values in Table I were then inserted into Eq. (1) to solve for the $S$ numbers. The particular noise range for the plots was selected because it conforms with the $S$ numbers commonly observed in clinical images. The lines in Fig. 2 are drawn through the mean $S$-number values for the two or three phantom thicknesses employed at each $\mathrm{kVp}$. The data in Fig. 2 are replotted in Fig. 3 to demonstrate the variation in $S$ number as a function of $\mathrm{kVp}$ for fixed noise $(\sigma)$ levels of $8,9,10$, and 11. As in Fig. 2, the lines in Fig. 3 pass through the mean $S$ numbers for the two or three phantom thicknesses at each $\mathrm{kVp}$.

The data in Fig. 3 show that for a particular $\mathrm{kVp}$ and noise level, the $S$ number does not vary significantly with the phantom thicknesses used. This result indicates that the $S$ number and the noise level will be useful for setting up phototimers.

\section{DISCUSSION}

The values of the exponent $b(-0.39$ to -0.44$)$ in Eq. (3), given in Table II, indicate that the standard deviation of the pixel values in the CR image is approximately inversely proportional to the square root of the $\mathrm{x}$-ray exposure at the IP. This is the expected relationship for a quantum-noise-limited system, such as CR. ${ }^{10,11}$ For CR, this expected relationship can be derived as follows: The pixel values in a Fuji CR image can be expressed as a function of the energy fluence absorbed in the IP, as ${ }^{12}$

$$
Q=\frac{1024}{L} \log \left(\frac{S}{C_{0}} A E_{\mathrm{ab}}\right)+511,
$$

\begin{tabular}{|c|c|c|c|c|c|}
\hline \multirow[b]{2}{*}{$\mathrm{kVp}$} & \multirow{2}{*}{$\begin{array}{l}\text { Lucite thickness } \\
\text { (in.) }\end{array}$} & \multicolumn{2}{|c|}{ With grid } & \multicolumn{2}{|c|}{ Without grid } \\
\hline & & $a$ & $b$ & $a$ & $b$ \\
\hline \multirow[t]{2}{*}{70} & 4 & 7.26 & -0.43 & 7.62 & -0.44 \\
\hline & 6 & 7.10 & -0.40 & 7.55 & -0.43 \\
\hline \multirow[t]{3}{*}{90} & 4 & 7.34 & -0.42 & 7.46 & -0.40 \\
\hline & 6 & 7.37 & -0.43 & 7.55 & -0.40 \\
\hline & 8 & 7.36 & -0.40 & 7.47 & -0.42 \\
\hline \multirow[t]{2}{*}{125} & 6 & 7.88 & -0.43 & 7.34 & -0.40 \\
\hline & 8 & 8.03 & -0.43 & 7.54 & -0.39 \\
\hline
\end{tabular}

TABLE II. Dependence of the values of the parameters $a$ and $b$ in equation $\sigma=a(\text { average exposure })^{b}$, on $\mathrm{kVp}$ and phantom thickness. 
WITH GRID

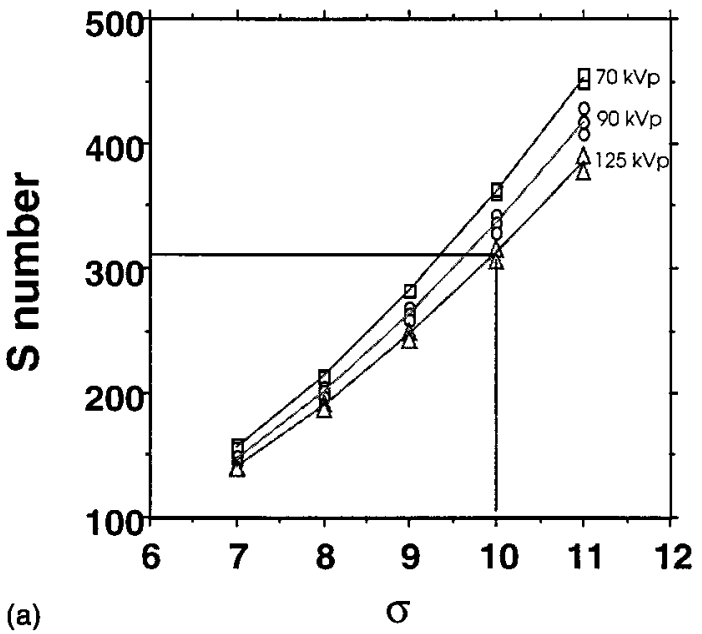

(a)

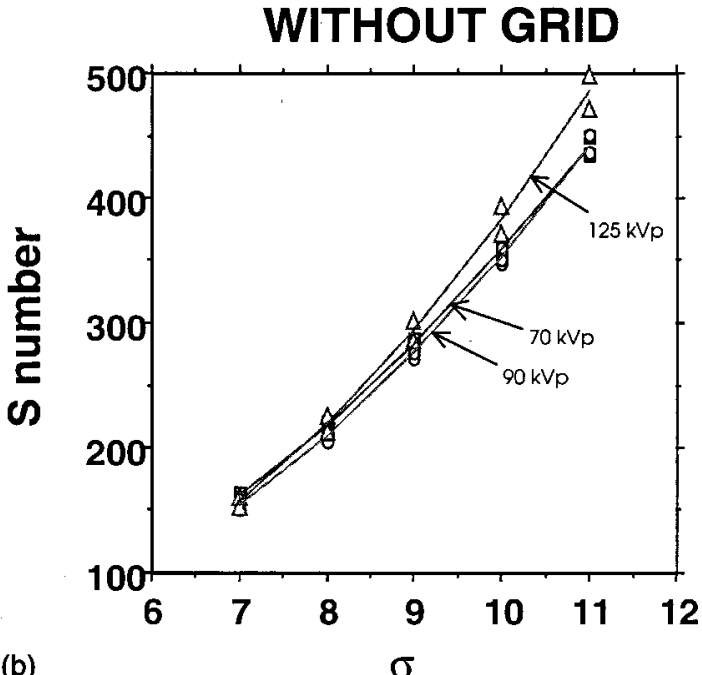

(b)

FIG. 2. Variation of the $S$ number with noise level $(\sigma)$ (a) with grid, and (b) without grid, for different $\mathrm{kVp}$ values (represented by different symbols). The data points of the same symbol at a given $\sigma$ and constant $\mathrm{kVp}$ represent data for different phantom thicknesses (4 and 6 in. for $70 \mathrm{kVp}, 4,6$, and 8 in. for $90 \mathrm{kVp}$, and 6 and $8 \mathrm{in}$. for $125 \mathrm{kVp}$ ).

where $Q$ is the pixel value, $E_{\mathrm{ab}}\left(\mathrm{J} / \mathrm{cm}^{2}\right)$ is the energy fluence absorbed in the IP, $A\left(\mathrm{~cm}^{2}\right)$ is the pixel size, $C_{0}(\mathrm{~J})$ is an empirical calibration constant, $S$ is the system sensitivity $(S$ number), and $L$ (unitless) is the system read latitude. For a ROI in a "flat field" image, the energy fluence absorbed in the IP is proportional to the mean exposure, $\bar{E}$, at the IP. Taking this into account, the variance in the pixel values is given by

$$
\sigma_{Q}^{2}=\left(\frac{d Q}{d \bar{E}}\right)^{2} \sigma_{\bar{E}}^{2}
$$

where $(d Q / d \bar{E})$ from Eq. (5) is proportional to $1 / \bar{E}$. Since $(\bar{E})$ is Poisson distributed, $\sigma_{\bar{E}}^{2}$ is proportional to $\bar{E}$. Substituting these relationships into Eq. (6), and taking the square root, we find the standard deviation of the pixel values in the CR image, $\sigma_{Q}$, should be proportional to $\bar{E}^{-1 / 2}$.

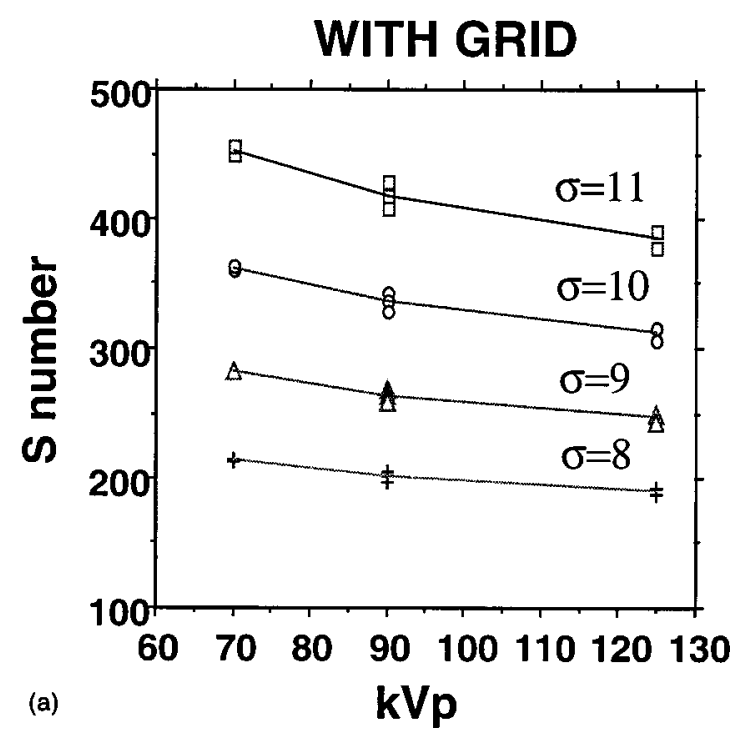

WITHOUT GRID

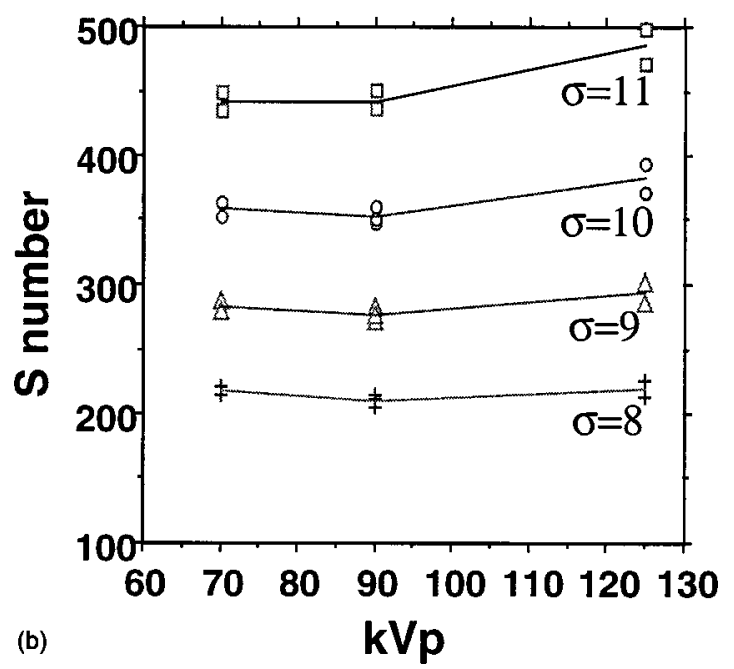

FIG. 3. Variation of the $S$ number with $\mathrm{kVp}$ (a) with grid, and (b) without grid, for noise levels $\sigma$ of the image that range from 8 to 11 (represented by different symbols). The data points of the same symbol at a given $\mathrm{kVp}$ and constant $\sigma$ represent data for different phantom thicknesses (4 and 6 in. for $70 \mathrm{kVp}, 4,6$, and $8 \mathrm{in}$. for $90 \mathrm{kVp}$, and 6 and 8 in. for $125 \mathrm{kVp}$ ).

The noise sources that contribute to the overall noise in a CR image are: $x$-ray quantum mottle, variation in the photostimulable luminescence of the imaging plate, electronic noise from the digitization process, ${ }^{13}$ and structured noise (from nonrandom sources such as nonuniformities in the phantom, grid defects, nonuniform response of the CR plate due to physical or chemical nonuniformities (e.g., variations in thickness, density, or number of electron traps at different locations on the plate, etc.) We performed a study to investigate whether the structural noise could account for the deviation of the measured values of the exponent $b(-0.39$ to $-0.44)$, from the expected value of -0.5 . Two images of the 6-in.-thick phantom were acquired using identical exposure technique. The images were then aligned and subtracted. Since the structured noise is the same in each image, it subtracted out, leaving only the noise due to quantum and elec- 
tronic effects. The standard deviation of the pixel values was measured in the subtracted image, and was divided by $\sqrt{ } 2$ to account for the increased noise due to the subtraction process. The exponent $b$ was calculated and found to be -0.49 . Thus, the deviation of $b$ from the expected value of -0.5 was found to be due to structured noise. For the practical purposes of setting up phototimers with CR, however, the exact determination of $\sigma$ according to Eq. (3) is not necessary.

For each $\mathrm{kVp}$, an $S$ number can be associated with the standard deviation of the pixel values that will produce an image with the desirable noise characteristics [Figs. 3(a) and $3(b)]$. The detectability of low-contrast objects in a digital image is determined mainly by its signal-to-noise ratio (SNR), which can be defined as the difference in the average pixel values between a low contrast object and its surrounding background divided by $\sigma$. For a given imaging condition, there is a threshold SNR below which the low contrast object cannot be detected. Therefore the correspondence between the $S$ number and $\sigma$ can be used to estimate the upper limit of the $S$ number that will allow low-contrast objects of a predetermined pixel value difference to be visible. For example, assuming a threshold SNR of 5, a pixel value difference of 50 will be visible when $\sigma$ is equal to or less than 10 ( $\sigma$ $\leqslant 50 / 5$ ). As can be seen from Fig. 2(a), this corresponds, in the case of $125 \mathrm{kVp}$ with grid, to an $S$ value of about 310 or less. Accordingly, the detection of yet smaller pixel value differences, or contrast $<50$, will require lower noise, smaller $S$ number, or higher exposure.

The exact $S$ number or $\sigma$ to employ for a particular examination depends on what a radiologist determines as an acceptable noise level at reasonable radiation dose, exposure time, and $\mathrm{x}$-ray tube loading. Radiologists in our department have historically chosen to employ 400 speed screen-film systems for general radiography and 200 speed screen-film systems for chest radiography. (Thus, they are willing to accept more noise in general imaging than in chest imaging.) Using this as a basis, we have adjusted the phototimers on our x-ray units for CR imaging so that the $S$ number is 400 $\pm 15 \%$ for general imaging exposure techniques of $50-90$ $\mathrm{kVp}$, and $200 \pm 15 \%$ for chest radiography at $125 \mathrm{kVp}$. The radiologists generally find the image quality resulting from these choices to be acceptable. A similar range (125-275) for the $S$ number of portable chest examinations was reported as acceptable by Seibert et $a l^{5}$

When setting up phototimers for screen-film radiography, a single cassette is employed in order to eliminate variability due to different screen response. Similarly, when setting up phototimers for CR, a single CR plate should be employed. For best results, this plate should be one that has an average sensitivity (i.e., a plate that produces an $S$ number that is close to the mean of the $S$ numbers for the entire set of plates, when all are exposed to the same amount of radiation, e.g., $1 \mathrm{mR}$ at $80 \mathrm{kVp}$.)

It is interesting to note that for all $\mathrm{kVp}$ and phantom thickness conditions studied without the grid, the constant $c$ in Eq. (1) (see Table I) is within about $8 \%$ of the value of 200 specified by Fuji at $80 \mathrm{kVp}$. However, with the grid at
70 to $90 \mathrm{kVp}, c$ is about 142, and at $125 \mathrm{kVp}, c$ is about 163 . This variability in $c$ and the corresponding difference in the $S$ number for a given $\sigma$, between the with-grid and withoutgrid conditions, indicate that it may be necessary to perform separate calibrations of the phototimers for the with-grid and without-grid conditions. Separate calibration is sometimes also needed when using a screen-film system as the detector.

Automatic exposure control (AEC) setup for screen-film involves calibration of the phototimer at various $\mathrm{kVp}$ 's to compensate for variations in the energy response of the screen-film system. These variations can be fairly large. For example, the speed of a terbium activated gadolinium oxysulfide $\left(\mathrm{Gd}_{2} \mathrm{O}_{2} \mathrm{~S}: \mathrm{Tb}\right)$ screen-film system is about $30 \%$ faster at 90 than at $70 \mathrm{kVp} .{ }^{14}$ From Fig. 3, the corresponding sensitivity variation for $\mathrm{CR}$ is much less at these $\mathrm{kVp}$ 's. Without a grid, it is insignificant, and with a grid, the sensitivity, as represented by the $S$ number, is at most about $7 \%$ lower at 90 than at $70 \mathrm{kVp}$. This smaller variation in response with $\mathrm{kVp}$ should make AEC setup easier with CR than with screenfilm systems.

Most clinical examinations with the Fuji CR system are performed using the Automatic mode to read the IPs. In the Automatic mode the reading sensitivity ( $S$ number) is determined by analyzing the image histogram from the entire imaging plate, as opposed to a predetermined subregion of the imaging plate in the Semi-automatic mode. To demonstrate the validity of setting up the phototimers using the semiautomatic mode, in a clinical situation where most images are processed using the auto mode, we evaluated a PA chest examination, which is one of the most common radiographic procedures. We employed a Picker MTX radiographic unit, for which the wall Bucky phototimers had been calibrated using the procedure described previously. Using a phototimer technique of $125 \mathrm{kVp}$ and $300 \mathrm{~mA}$ we separately imaged a Duke University Chest Phantom ${ }^{15}$ (Nuclear Associates Model 07-646 QC Phantom for Digital Chest Radiography), and a 5-in.-thick slab of Lucite. The latter was chosen because it results in about the same phototimed $\mathrm{mA} \mathrm{s}$ as the chest phantom. The entrance exposure was also monitored by placing a $15 \mathrm{cc}$ Keithley ionization chamber on the entrance surface of the chest phantom, and on the entrance surface of the $5 \mathrm{in}$. of Lucite. In both cases the ionization chamber was positioned away from the location of the phototimers. A CR cassette loaded with an IP was positioned in the wall Bucky. Ten exposures were made for each phantom using ten different IPs. For the chest phantom the resulting technique was $5.3 \pm 0.1 \mathrm{~mA} \mathrm{~s}$ and the entrance exposure was $29.0 \pm 0.4 \mathrm{mR}$. The images of this phantom were processed using the "PA Chest" submenu, which is used routinely for the corresponding clinical examinations. In this submenu the $S$ number is determined using the auto mode. The range of $S$ numbers for the ten randomly selected IPs, from the pool of the IPs used clinically, was 205 \pm 22 . For the 5 in. of Lucite the resulting technique was $4.6 \pm 0.1 \mathrm{mAs}$ and the entrance exposure was $30.6 \pm 0.7 \mathrm{mR}$. The images, for this homogeneous phantom, were processed using the "Test-Sensitivity" menu in which the $S$ number is determined using the semiauto mode. The range of $S$ numbers for the same ten IPs used 


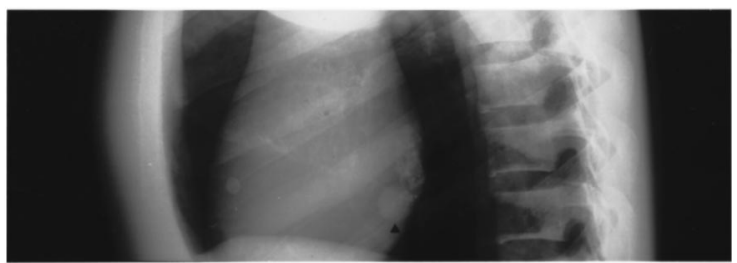

(a)

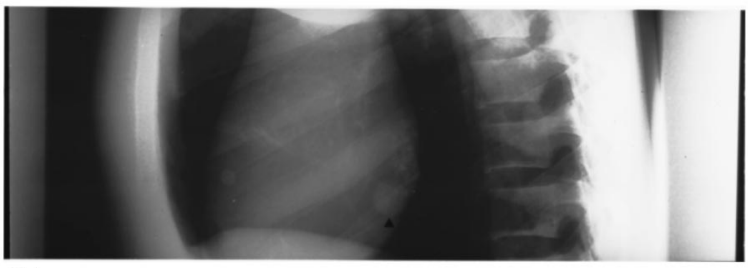

(b)

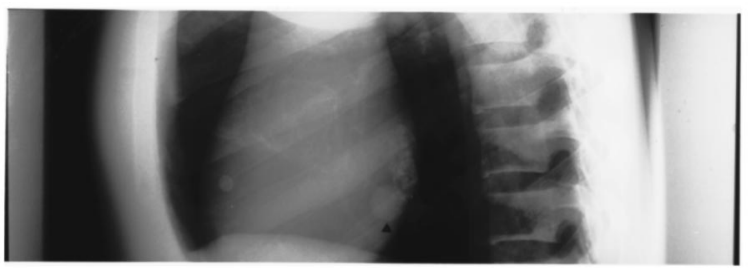

(c)

FIG. 4. CR image of the lateral view of an anthropomorphic chest phantom (a) without $\mathrm{x}$-ray beam collimation, (b) with the $\mathrm{x}$-ray beam tightly collimated around the phantom contour and processed with the same parameters as the image in (a), and (c) the image in (b) after reprocessing (GS increased by 100$)$.

to image the chest phantom was $185 \pm 13$. This demonstrates clearly that after the phototimers have been calibrated for flat fields using the semi-auto mode of the "Test-Sensitivity" menu (for chest exams $S$ number set to $200 \pm 40$ ), the resulting clinical images, using the auto mode of the "Chest-PA Chest' menu, are very similar both from the radiologists' perspective ( $S$ numbers $185 \pm 13$ vs $205 \pm 22$ ) as well as from the perspective of patients' exposure $(30.6 \pm 0.7$ vs 29.0 $\pm 0.4 \mathrm{mR}$ ). Although this was demonstrated using a chest phantom, similar results are obtained with patient images.

The histogram for the Automatic mode excludes regions corresponding to the collimator and direct exposure. These regions are automatically detected by some fairly robust algorithms. However, these algorithms sometimes fail and a significantly different $S$ number may be obtained in certain cases that have the same skin entrance exposure. Thus, the $S$ number may not necessarily represent the exposure to the plate. An example of such a case is shown in Fig. 4. Figure 4(a) shows the lateral view of an anthropomorphic chest phantom, without $\mathrm{x}$-ray beam collimation. This image was processed using the Automatic mode and the "Lateral Chest" processing algorithm that are used routinely in our clinic. The $S$ number is 132 , the mean pixel value in a selected area of the image (indicated by the arrow) is 278 , and $\sigma$ is 40. Figure 4(b) shows a similar CR image in which the $\mathrm{x}$-ray beam is tightly collimated around the phantom contour. For the same patient exposure and processing parameters, the $S$ number is 252 , which is nearly a factor of 2 greater, im- plying half the plate exposure. The mean pixel value in the same selected area of the image is $70, \sigma$ is 20 , and the image appears overall "darker." CR images such as the one displayed in Fig. 4(b) can sometimes be reprocessed using a different transfer curve to recover image detail. Figure 4(c) shows the result of reprocessing the image in Fig. 4(b), by increasing the gradation shift parameter, GS, by 100 (equivalent to changing the speed of a screen-film system.) The image now looks very similar to the one in Fig. 4(a). The mean pixel value in the selected area of the image is 261, and the standard deviation of the pixel values is 37 . Both of these are comparable to the values obtained for the uncollimated image in Fig. 4(a).

To investigate the magnitude of the change in $S$ number for the images in Figs. 4(a) and 4(b) that may be due to the reduced scatter for the smaller field size condition, we used 6 in. of Lucite at $90 \mathrm{kVp}$ and compared the $S$ numbers obtained with a full field, and with fields collimated to $80 \%$ and $60 \%$. The $S$ numbers obtained were 142,149 , and 152 , respectively. This indicates that the effect of the reduced scatter on the $S$ number is minimal, and that the differences between Figs. 4(a) and 4(b) are mainly due to the processing algorithm. Sometimes, however, recovery of the image by reprocessing may not be achievable. This can be a particularly important problem in pediatric cases where collimation is used more often.

\section{CONCLUSIONS}

Our studies indicate that the $S$ number in the Fuji CR system is closely related to the low contrast sensitivity of the system. A similar relationship is expected for the corresponding plate exposure indicator values for other CR systems. The plate exposure indicator value and the standard deviation $(\sigma)$ of the pixel values in a "flat field" image can thus be used to setup phototimers. At our institution, where 400-speed screen-film is used for general radiography and 200 speed for chest radiography, radiologists generally find CR image quality acceptable when $\sigma \leqslant 11(S \leqslant 400)$ for general radiography $(50-90 \mathrm{kVp})$, and $\sigma \leqslant 8(S \leqslant 200)$ for chest radiography $(125 \mathrm{kVp})$. However, one must be cautious when assessing image quality from the plate exposure indicator value of a clinical image, since factors other than the amount of x-ray quanta that form the useful image, such as the image processing mode and the amount of collimation, may affect both the plate exposure indicator value and the image quality.

\footnotetext{
a) Author to whom all correspondence should be addressed; electronic mail: manos@umich.edu

${ }^{1} \mathrm{M}$. Sonoda et al., " Computed radiography utilizing scanning laser stimulated luminescence,” Radiology 148, 833-838 (1983).

${ }^{2}$ H. Kato, "Photosimulable phosphor radiography design considerations," in Specification, Acceptance Testing and Quality Control of Diagnostic $X$-ray Imaging Equipment, edited by J. A. Seibert, G. T. Barnes, and R. G. Gould (AIP, Woodbury, NY, 1994), pp. 731-770.

${ }^{3}$ J. A. Seibert, "Photostimulable phosphor system acceptance testing," in Medical Physics Monograph No. 20: Specification, Acceptance Testing and Quality Control of Diagnostic X-ray Imaging Equipment (AIP, Washington, DC, 1994), pp. 771-880.
} 
${ }^{4}$ S. S. Sagel et al., "Digital mobile radiography," J. Thorac. Imaging $\mathbf{5}$, 36-48 (1990)

${ }^{5}$ J. A. Seibert, D. K. Shelton, and E. H. Moore, "Computed radiography x-ray exposure trends," Acad. Radiol. 3, 313-318 (1996).

${ }^{6}$ Fuji Computed Radiography, Technical Review No. 3, "Automatic Setting Functions for Image Density and Range in the FCR System," Fuji Photo Film Co. Ltd., Tokyo, Japan, 1993.

${ }^{7} \mathrm{~T}$. Bogucki (private communication).

${ }^{8} \mathrm{C}$. Willis (private communication).

${ }^{9} \mathrm{C}$. Willis and J. Lobick, "Updating the Agfa component of the AAPM task group \#10 draft report on computed radiography acceptance testing and quality control," Med. Phys. 25, 1587 (1998).

${ }^{10} \mathrm{~W}$. Hillen, U. Schiebel, and T. Zaengel, "Imaging performance of a digital storage phosphor system," Med. Phys. 14, 744-751 (1987).

${ }^{11} \mathrm{H}$. Fujita et al., "Basic imaging properties of a computed radiographic system with photostimulable phosphors,' Med. Phys. 16, 52-59 (1989).

${ }^{12}$ D. M. Tucker and P. S. Rezentes, "The relationship between pixel value and beam quality in photostimulable phosphor imaging," Med. Phys. 24, 887-893 (1997).

${ }^{13} \mathrm{G}$. T. Barnes, "Digital x-ray image capture with image intensifier and storage phosphor plates: Imaging principles, performance and limitations," in Digital Imaging, edited by W. R. Hendee and J. H. Trueblood (Medical Physics Publishing, Madison, WI, 1993), pp. 25-48.

${ }^{14}$ T. S. Curry, J. E. Dowdey, and R. C. Murry, Christensen's Physics of Diagnostic Radiology, 4th ed. (Lea and Febiger, Philadelphia, 1990), pp. 130 .

${ }^{15}$ H. G. Chotas, C. E. Floyd, G. A. Johnson, and C. E. Ravin, "Quality control phantom for digital chest radiography," Radiology 202, 111-116 (1997). 